\title{
Caracterização físico-química e determinação de coeficientes cinéticos aeróbios de remoção da matéria orgânica de águas residuárias agroindustriais
}

\author{
Physical-chemical characterization and determination of aerobic kinetic \\ coefficients for the removal of organic matter from agro-industrial wastewater \\ Naassom Wagner Sales Morais ${ }^{1} \oplus$, Milena Maciel Holanda Coelho ${ }^{2} \odot$, \\ Francisco Schiavon Souza Silva' ${ }^{\oplus}$, Erlon Lopes Pereira' ${ }^{\circledR}$, André Bezerra dos Santos ${ }^{1 *}$ ()
}

\begin{abstract}
RESUMO
Os objetivos do presente trabalho foram realizar uma caracterização físicoquímica de diferentes águas residuárias agroindustriais (ARA) e aplicar modelos cinéticos de primeira e de segunda ordem para verificar qual deles descreve melhor a progressão da demanda bioquímica de oxigênio (DBO) e para determinar coeficientes cinéticos aeróbios de remoção da matéria orgânica das ARA. Efetuou-se o ensaio de progressão da DBO pela incubação de ARA em um sistema respirométrico (Oxitop ${ }^{\circledR}$ ). Os modelos cinéticos de primeira e de segunda ordem foram avaliados por erro quadrático médio (RMSE), erro quadrático médio normalizado (NRMSE) e critério de informação de Akaike (AIC). A cinética de biodegradação aeróbia das ARA avaliadas ajustou-se melhor ao modelo de primeira ordem em termos de DBO total e solúvel. Na progressão de DBO total, o maior coeficiente de desoxigenação de primeira ordem $(K)$ foi o da água residuária de abatedouro - ARB $\left(0,56 d^{-1}\right)$ - e o menor foi o da água residuária de suinocultura - ARS $\left(0,16 d^{1}\right)$. Os coeficientes de desoxigenação determinados no presente trabalho mostraram-se representativos, podendo ser utilizados para a simulação de processos de degradação da matéria orgânica em condições aeróbias.
\end{abstract}

Palavras-chave: caracterização físico-química; constante cinética de desoxigenação; águas residuárias agroindustriais; modelagem cinética.

\begin{abstract}
The aim of the present work was to perform a physicochemical characterization of different agroindustrial wastewaters (AIW), to apply first and second order kinetic models to verify which one best describes the progression of the biochemical oxygen demand (BOD) and to determine the aerobic kinetics coefficients of organic matter removal from AIW. The BOD progression assay was performed from the incubation of AIW in a respirometric system (Oxitop ${ }^{\circledR}$ ). The first and second order kinetic models were evaluated by mean square error (RMSE), normalized mean square error (NRMSE), and Akaike Information Criterion (AIC). The aerobic biodegradation kinetics of the AIW evaluated was better fitted to the first order model in terms of total and soluble BOD. In the total BOD progression, the highest coefficient of first-order deoxygenation ( $k^{\prime}$ ) was that of slaughterhouse wastewater (SW; $0.56 \mathrm{~d}^{-1}$ ) and the lowest was that of swine wastewater (SSW; $0.16 d^{-1}$ ). The deoxygenation coefficients determined in the present work were representative, and can be used for the simulation of degradation processes of organic matter under aerobic conditions.
\end{abstract}

Keywords: physical-chemical characterization; kinetic deoxygenation constant; agroindustrial wastewater; kinetic modeling.

\section{INTRODUÇÃO}

A agroindústria é um dos principais setores produtivos do agronegócio que gera impactos socioeconômicos positivos no Brasil. Em 2017, segundo dados do Centro de Estudos Avançados em Economia Aplicada da Universidade de São Paulo (CEPEA/USP) e da Confederação da
Agricultura e Pecuária do Brasil (CNA), o agronegócio correspondeu a cerca de $21,6 \%$ do produto interno bruto (PIB) nacional, com alta de 2,7\% da agroindústria (CEPEA, 2018).

Em um panorama internacional, nas últimas três décadas, a agroindústria cresceu rapidamente. Na União Europeia, a agricultura 
ocupa em torno de $45 \%$ do uso da terra e mais de $30 \%$ do uso total da água (BOSONA, GEBRESENBET, 2018; TAMBURINI et al., 2015). Conforme a Organização das Nações Unidas para a Alimentação e a Agricultura dos Estados Unidos (FAO, 2017), a agroindústria corresponde a mais de $50 \%$ do valor agregado da manufatura em países de baixa renda e a $30 \%$ em países de renda média. Estima-se que, no período de 1980-2005, os países em desenvolvimento dobraram sua participação em produtos de valor agregado pela manufatura de alimentos, bebidas e tabaco.

Nessa perspectiva, o contínuo crescimento da agroindústria significa a crescente geração de resíduos orgânicos, como as águas residuárias agroindustriais (ARA), durante os processos de transformação da matéria-prima e de atividades relacionadas. Entre as ARA que são geradas em altos volumes e em diferentes agroindústrias brasileiras, podem ser citadas as geradas nas agroindústrias de produção de carne/abatedouro (ARB), de suinocultura (ARS), de cerveja (ARC), de laticínios e derivados (ARL) e de beneficiamento de frutas para produção de sorvetes (ARBF).

O descarte inadequado desses resíduos orgânicos no meio ambiente pode ocasionar a poluição e a contaminação do solo, da água e do ar, a proliferação de vetores de doenças, disfunções hormonais na fauna aquática e terrestre pela presença de micropoluentes e a promoção de resistência bacteriana como resultado da presença de antibióticos (CHENG et al., 2019). O lançamento de ARA in natura em corpos hídricos promove a alteração das características físico-químicas da água, favorece a proliferação de algas e de organismos patogênicos e o consumo de oxigênio dissolvido, ocasionando a diminuição da qualidade do manancial e a redução dos usos múltiplos das águas (HILARIO GARCIA et al., 2017; XIAO et al., 2018).

Ante isso, para a conservação dos recursos naturais, o tratamento desse resíduo líquido industrial é fundamental. Considerando que a maior parte da matéria orgânica presente nas ARA é biodegradável, muitas estações de tratamento de águas residuárias projetadas contemplam tratamentos biológicos anaeróbios e aeróbios (MENKITI et al., 2014). Alguns arranjos consistem em tratamento preliminar, tratamento anaeróbio em reatores de alta taxa, como o reator anaeróbio de manta de lodo e fluxo ascendente (UASB), o reator anaeróbio de leito granular expandido (EGSB) e o reator de circulação interna (IC), seguido de um pós-tratamento aeróbio, como lodo ativado ou filtros aerados, e unidades de polimento final, que variam conforme os usos que se queira dar à água residuária tratada. Muitas estações ainda são projetadas com sistemas de lodo ativado em fluxo contínuo seguido de um polimento final, apesar dos reconhecidos maiores custos de implantação, operação e manutenção, se comparados aos do processo anaeróbio-aeróbio (CHAN et al., 2009).

A demanda bioquímica de oxigênio (DBO) exercida no tempo está relacionada ao coeficiente de desoxigenação $(k)$ que pode ser obtido em laboratório (MATOS et al., 2017). Contudo há divergência na literatura sobre qual dos modelos melhor descreve a DBO exercida no tempo. May (1971) afirma que a soma das progressões da degradação de substratos simples remeteria a curvas de cinética de primeira ordem, e, portanto, essa equação seria mais adequada para descrever a progressão de DBO no tempo.

De fato, para muitos estudos, os modelos determinísticos de primeira ordem representam um equilíbrio entre a simplicidade e a precisão, e, por esse motivo, a cinética de primeira ordem é a mais aceita para descrever a progressão de DBO exercida no tempo (BHATT et al., 2016; SIBIL; BERKUN; BEKIROGLU, 2014). Já alguns autores (ADRIAN; SANDERS, 1998; ZANONI, 1967) afirmam que o modelo de segunda ordem seria o mais adequado para descrição da DBO no tempo do que o modelo de primeira ordem, visto que a soma das progressões do consumo de cada composto presente na água residuária é algo de natureza complexa.

Em busca de uma melhor compreensão do processo de degradação da matéria orgânica em condições aeróbias, a aplicação da modelagem cinética para descrever a taxa de degradação de efluentes agroindustriais é de suma importância para o fornecimento de constantes cinéticas utilizadas no projeto de estações de tratamento, contudo ainda são escassos os trabalhos que apresentem a cinética do processo aeróbio, especialmente para ARA.

Ante o exposto, os objetivos do presente trabalho foram realizar uma caracterização físico-química de diferentes ARA e aplicar modelos cinéticos de primeira e de segunda ordem para verificar qual deles descreve melhor o processo de progressão da demanda bioquímica de oxigênio (DBO) e para determinar coeficientes cinéticos aeróbios de remoção da matéria orgânica das ARA.

\section{MATERIAL E MÉTODOS}

\section{Substratos e caracterização físico-química}

As águas residuárias agroindustriais (ARA) utilizadas neste experimento foram: água residuária de abatedouro (ARB), de suinocultura (ARS), de cervejaria (ARC), de laticínios (ARL) e de beneficiamento de frutos (ARBF) da indústria de sorvetes. As ARA foram coletadas em diferentes municípios do estado do Ceará, Brasil.

Após a coleta, preservaram-se as amostras a $3^{\circ} \mathrm{C}$, visando minimizar a atividade microbiana, manter as características físico-químicas das amostras e reduzir o risco de degradação da matéria orgânica durante $\mathrm{o}$ armazenamento/transporte. $\mathrm{O}$ tempo entre a coleta e o preparo das amostras para a realização dos ensaios de $\mathrm{DBO}$ não foi superior a $6 \mathrm{~h}$ (MATOS et al., 2017). A caracterização físico-química das ARA in natura foi realizada de acordo com o Standard Methods for the Examination of Water and Wastewater (APHA, 2012). 


\section{Modelagem cinética da progressão de demanda bioquímica de oxigênio e determinação dos coeficientes cinéticos de desoxigenação}

O ensaio de progressão de DBO exercida no tempo utilizou um sistema respirométrico (Oxitop ${ }^{\circledR}$, Velp Scientifica, Estados Unidos), constituído de um microprocessador sem mercúrio que permitia a leitura da pressão exercida dentro dos frascos, convertendo-a em DBO. Isso porque os microrganismos aeróbios presentes no meio consumiam o oxigênio do headspace para degradar a matéria orgânica das ARA. O sensor do aparelho registrava a pressão interna dentro do frasco que era proporcional ao material orgânico das ARA.

O sistema Oxitop ${ }^{\circledast}$ era composto de garrafas de vidro escuras de $500 \mathrm{~mL}$, com suporte de álcali acoplado ao bocal das garrafas, no qual também era rosqueado, e pelo sensor BOD Sensor System 6, que realizava uma amostragem cumulativa do valor da DBO exercida a cada $24 \mathrm{~h}$, sendo o valor médio registrado.

O meio basal foi feito de acordo com o procedimento de incubação 5210B da metodologia de análise de DBO do Standard Methods for the Examination of Water and Wastewater (APHA, 2012). O meio basal e a amostra de ARA mantiveram-se homogeneizados utilizando uma barra de agitação e um agitador VELP MST durante todo o processo de incubação e mantidos em estufa BOD (TE-401, Tecnal LTDA, Brasil) sob temperatura de $20^{\circ} \mathrm{C}$ por tempo suficiente até a estabilização da leitura e, portanto, estabilização do consumo da matéria orgânica. Para a realização dos ensaios de DBO solúvel, filtraram-se as amostras em membrana de fibra de vidro com poro de 0,45 $\mu \mathrm{m}$ (EMD Millipore, Estados Unidos).

Os modelos de primeira e de segunda ordem que descrevem a progressão da $\mathrm{DBO}$ total $\left(\mathrm{DBO}_{\mathrm{T}}\right)$ e $\mathrm{DBO}$ solúvel $\left(\mathrm{DBO}_{\mathrm{S}}\right)$ por meio do consumo de oxigênio dissolvido (DBO exercida ao longo do tempo) e estimam os coeficientes de desoxigenação de primeira $(k$ ') e de segunda ordem ( $k$ "), respectivamente, são apresentados nas Equações 1 e 2.

$D B O_{t}=D B O_{u} \cdot\left(1-e^{-k^{\prime} \cdot t}\right)$

$D B O_{t}=\frac{k^{\prime \prime} \cdot\left(D B O_{u}\right)^{2} \cdot t}{1+k^{\prime \prime} \cdot D B O_{u} \cdot t}$

Em que:

$\mathrm{DBO}_{\mathrm{t}}=\mathrm{DBO}$ exercida em um tempo $t$ qualquer $\left(\mathrm{mg} \mathrm{O}_{2} \mathrm{~L}^{-1}\right)$;

$\mathrm{DBO}_{\mathrm{U}}=\mathrm{DBO}$ última $\left(\mathrm{mg} \mathrm{O}_{2} \mathrm{~L}^{-1}\right)$;

$k^{\prime}=$ coeficiente cinético de desoxigenação de primeira ordem $\left(\mathrm{d}^{-1}\right)$;

$k$ " = coeficiente cinético de desoxigenação de segunda ordem

$\left(\mathrm{L} \mathrm{g} \mathrm{O}_{2}^{-1} \mathrm{~L}^{-1} \mathrm{~d}^{-1}\right)$;

$t=$ tempo $(\mathrm{d})$.
Os modelos de primeira e de segunda ordem foram ajustados aos dados experimentais por meio de análise de regressão não linear de mínimos quadrados usando a ferramenta solver do Microsoft Excel 2013. Assim, estimaram-se os parâmetros dos modelos cinéticos ( $k^{\prime}$ e $k^{\prime \prime}$ ). Esse método ajusta valores das variáveis do modelo visando minimizar a soma dos quadrados das diferenças entre os valores previstos e os medidos. Conforme Oke e Akindahunsi (2005), a regressão não linear é o método mais preciso para estimar o valor de $k$. Para determinar a correlação entre os valores experimentais e preditos pelos modelos, calculou-se o coeficiente de determinação $\left(\mathrm{R}^{2}\right)$ conforme Equação 3:

$R^{2}=1-\frac{\sum_{i}\left(Y_{i, \exp }-Y_{i, \text { est }}\right)^{2}}{\sum_{i}\left(Y_{i, \exp }-\bar{Y}\right)^{2}}$

Em que:

$\mathrm{Y}_{\mathrm{i}, \mathrm{exp}}=\mathrm{o}$ valor de dados experimentais;

$\mathrm{Y}_{\mathrm{i}, \mathrm{est}}=\mathrm{o}$ valor estimado pelo modelo;

$\overline{\mathrm{Y}}=$ a média dos dados experimentais.

A seleção do modelo que descreve melhor a progressão de DBO de cada ARA foi realizada usando as seguintes funções de erro: erro quadrático médio (RMSE), erro quadrático médio normalizado (NRMSE) e critério de informação de Akaike (AIC) (AKAIKE, 1998). Segundo Emiliano et al. (2009), o AIC é um dos métodos mais adequados e utilizados para a seleção e a comparação de modelos por uma pequena base de dados. Quanto menor o valor dos erros, maior a adequação dos dados estimados pelo modelo cinético aos dados experimentais. O RMSE, o NRMSE e o AIC foram calculados conforme Equações 4, 5 e 6, respectivamente.

$R M S E=\sqrt{\frac{\sum_{i}\left(Y_{i, \exp }-Y_{i, \text { est }}\right)^{2}}{n}}$

NRMSE $=\left[\frac{R M S E}{\left(Y_{\max }-Y_{\min }\right)}\right] \times 100$

$A I C=N \cdot \ln \left(\frac{S S}{N}\right)+2 k$

Em que:

$n=$ o número de pontos de dados experimentais (observações);

$\mathrm{Y}_{\max }$ e $\mathrm{Y}_{\min }=$ os valores máximo e mínimo observados para a variável resposta avaliada;

AIC = o critério de informação de Akaike (adimensional);

$\mathrm{N}=$ o número de observações de dados experimentais;

$S S$ = a soma quadrada dos resíduos;

$k=$ o número de parâmetros do modelo. 


\section{RESULTADOS E DISCUSSÃO}

\section{Substratos e caracterização físico-química}

As ARA (ARB, ARS, ARC, ARL e ARBF) avaliadas usualmente apresentaram características bastante distintas em relação às frações orgânicas, aos nutrientes, aos sólidos e aos demais constituintes, assim como entre as naturezas dissolvida e particulada (Tabela 1), estando de acordo com vários relatos da literatura, como Demirel et al. (2013) para a ARBF; Enitan et al. (2015) e Corsino et al. (2017) para a ARC; Fia et al. (2015), Pereira, Paiva e Silva (2016) e Rajab et al. (2017) para a ARB; Pereira et al. (2011), Motteran, Pereira e Campos (2013b) e Cheng et al. (2018) para a ARS; Abreu et al. (2013), Ribeiro et al. (2017) e Daneshvar et al. (2019) para a ARL.

$\mathrm{A} A \mathrm{ARB}$ e a ARS apresentaram $\mathrm{pH}$ na faixa de neutralidade (6,8 e 7). A ARL e a ARBF apresentaram valores de $\mathrm{pH}$ baixo (5,4 e 5,5), o que pode favorecer a corrosividade e a agressividade na instalação hidráulica existente nos sistemas de tratamento dessas ARA, como: sistema de bombeamento, elevatórias, tubulações de sucção, recalque e condução desses resíduos. Já a ARC apresentou pH elevado, o que pode ocasionar incrustações na instalação hidráulica existente nos sistemas de tratamento desse efluente.

Além do $\mathrm{pH}$, as concentrações de ácidos voláteis totais (AVT) e alcalinidade total (AT) são parâmetros importantes para a manutenção do tamponamento durante os processos biológicos de tratamento, condições de precipitação química de metais e incrustação de tubulação. Como pode ser observado na Tabela 1, a ARS foi a que apresentou maior valor de AT $\left(2.932,1 \mathrm{mgCaCO}_{3} \mathrm{~L}^{-1}\right)$ e AVT $\left(2.932,1 \mathrm{mgH}_{3} \mathrm{COOH} \mathrm{L}{ }^{-1}\right)$ quando comparada aos demais resíduos, os quais se mantiveram na faixa de AT de 187,5 a 370,6 mgCaCO$_{3} \mathrm{~L}^{-1}$ e AVT de 212,7 a 583,2 mgH $_{3} \mathrm{COOH} \mathrm{L}^{-1}$, comumente encontrados em trabalhos que discutem a caracterização dessas tipologias de ARA.

Segundo Pereira, Campos e Motteran (2013), esses altos valores de AT e AVT para ARS ocorrem pelas constituintes da ração suína; os sais de amônio, magnésio e cálcio contribuem com as altas concentrações de AT e os carboidratos facilmente fermentescíveis utilizados pelo suíno como fonte de energia (50 a 60\% que compõem a ração) estão presentes nos dejetos já convertidos em ácidos pelo processo digestivo e também em constante conversão pela presença de bactérias na ARS, justificando os valores de AVT encontrados.

As concentrações de sólidos na água são afetadas por todos os contaminantes presentes, com exceção dos gases dissolvidos; a fração dissolvida (sólidos dissolvidos totais - SDT, sólidos dissolvidos voláteis - SDV, sólidos dissolvidos fixos - SDF) influencia na demanda química de oxigênio solúvel $\left(\mathrm{DQO}_{\mathrm{S}}\right)$, demanda bioquímica de oxigênio solúvel $\left(\mathrm{DBO}_{5}{ }^{20}{ }_{\mathrm{S}} \mathrm{C}\right)$, condutividade elétrica $(\mathrm{CE})$ e cor real das ARA. Já a fração suspensa (sólidos suspensos totais - SST, sólidos suspensos voláteis - SSV, sólidos suspensos fixos - SSF) influencia na turbidez da amostra, bem como na demanda química de oxigênio particulada
$\left(\mathrm{DQO}_{\mathrm{p}}\right)$, demanda bioquímica de oxigênio particulado $\left(\mathrm{DBO}_{5}{ }^{20^{\circ} \mathrm{C}} \mathrm{p}\right)$. $\mathrm{Na}$ ARB, ARS, ARC, ARL e ARBF, os sólidos orgânicos (STV) representam mais de $50 \%$ dos sólidos totais (ST). Para a ARB e a ARS, a maioria dos ST é encontrada na forma suspensa, justificando os altos valores de $\mathrm{DQO}_{\mathrm{P}}, \mathrm{DBO}_{5}{ }^{20^{\circ} \mathrm{C}} \mathrm{P}$. A presença de elevada quantidade de material particulado torna o tratamento mais complexo, visto que dificulta a assimilação biológica direta, exigindo hidrólise prévia aeróbia ou anaeróbia (MOTTERAN; PEREIRA; CAMPOS, 2013a; PEREIRA; PAIVA; SILVA, 2016; YANG et al., 2016). Já uma composição predominante de material dissolvido é observada para as ARC, ARL e ARBF.

Os óleos e graxas podem causar problemas operacionais no sistema de coleta nas estações de tratamento de esgoto (ETE). Nos sistemas de coleta, esses compostos podem se depositar no interior das tubulações, provocando restrições ao fluxo líquido, redução da velocidade do escoamento, elevação da altura da lâmina líquida e consequentes entupimentos (MOTTERAN; PEREIRA; CAMPOS, 2013a). Durante o tratamento biológico, os óleos e graxas ocasionam a redução da atividade biológica em consequência da aderência às células microbianas, o que dificulta a absorção de matéria orgânica. As ARA apresentaram concentrações de óleos e graxas entre 22,3 e 147,0 $\mathrm{mg} \mathrm{L}^{-1}$, estando abaixo do valor de $3.677,0 \mathrm{mg} \mathrm{L}^{-1}$ estabelecido por Cristovão et al. (2016) como limite para processos aeróbios.

A relação demanda bioquímica de oxigênio total $\left(\mathrm{DBO}_{5}{ }^{20^{\circ} \mathrm{C}} \mathrm{T}\right) /$ demanda química de oxigênio total $\left(\mathrm{DQO}_{\mathrm{T}}\right)$ indicou que $36,42,41$, 67 e $79 \%$ da matéria orgânica da ARB, ARS, ARC, ARL e ARBF, respectivamente, pode ser biodegradada aerobiamente, sugerindo que tais efluentes podem potencialmente ser tratados por processos biológicos aeróbios. Utilizando os resultados da Tabela 1, é possível calcular a relação DBO:N:P para cada ARA, obtendo as relações 147:2:1 para a ARC, 113:2:1 para a ARL, 61:3:1 para a ARBF, 209:26:1 para a ARB e 127:11:1 para a ARS, indicando que as ARC e ARL são pobres em nitrogênio em relação a sua quantidade de carbono natural; as ARS e ARB são ricas em carbono e nitrogênio; já a ARBF é pobre em carbono e nitrogênio.

Assim, para aperfeiçoar o estado da arte sobre processos biológicos aeróbios no tratamento de ARA, além da caracterização, é de extrema importância a obtenção da taxa de remoção da matéria orgânica, a qual é medida pelo coeficiente de desoxigenação. Tal parâmetro poderá ser utilizado para diminuir o valor do tempo de ciclo ou de detenção hidráulica dos reatores aeróbios, assim como auxiliar em estudos sobre a capacidade de autodepuração dos corpos aquáticos.

\section{Modelagem cinética da progressão de demanda bioquímica de oxigênio e determinação dos coeficientes cinéticos de desoxigenação}

Com exceção da ARBF, o modelo de primeira ordem foi o que melhor se ajustou à cinética de progressão de $\mathrm{DBO}_{\mathrm{T}}$ para as ARA estudadas, 
Tabela 1 - Caracterização físico-química das águas residuárias agroindustriais (ARA) in natura.

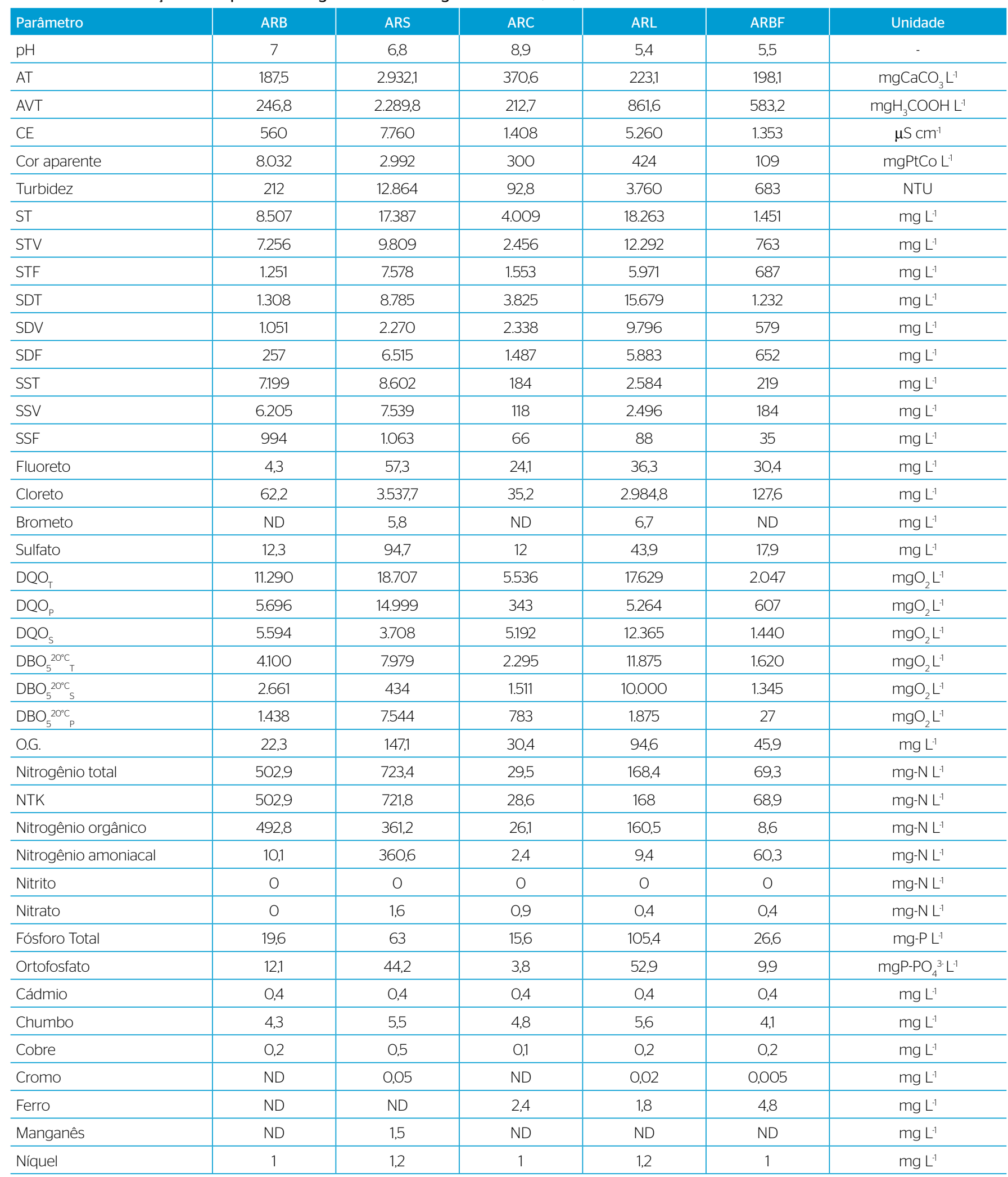

ARB: água residuária de abatedouro; ARS: água residuária de suinocultura; ARC: água residuária de cervejaria; ARL: água residuária de laticínios; ARBF: água residuária de beneficiamento de frutas para produção de sorvetes; pH: potencial hidrogeniônico; AT: alcalinidade total; AVT: ácidos voláteis totais; CE: condutividade elétrica; ST: sólidos totais; STV: sólidos totais voláteis; STF: sólidos totais fixos; SDT: sólidos dissolvidos totais; SDV: sólidos dissolvidos voláteis; SDF: sólidos dissolvidos fixos; SST: sólidos suspensos totais; SSV: sólidos suspensos voláteis; SSF: sólidos suspensos fixos; $\mathrm{DBO}$ : demanda bioquímica de oxigênio; $\mathrm{DQO}_{\mathrm{T}}$ : demanda química de oxigênio total; $\mathrm{DQO}_{\mathrm{S}}$ : demanda química de oxigênio solúvel; $\mathrm{DQO}_{\mathrm{p}}$ : demanda química de oxigênio particulado; O.G.: óleos e graxas; NTK: nitrogênio total Kjeldahl; ND: não detectado; $\mathrm{DBO}_{\mathrm{T}}$ : demanda bioquímica de oxigênio total; $\mathrm{DBO}_{\mathrm{S}}$ : demanda bioquímica de oxigênio solúvel; $\mathrm{DBO}_{\mathrm{p}}$ : demanda bioquímica de oxigênio particulada. 
visto que se obtiveram maiores valores do coeficiente de determinação e menores valores das funções de erro quando comparado ao modelo de segunda ordem, como pode ser observado pelos dados expressos na Tabela 2.

As progressões de $\mathrm{DBO}_{\mathrm{T}}$ e $\mathrm{DBO}_{\mathrm{S}}$ da $\mathrm{ARC}$ foram as que forneceram os melhores ajustes pelo modelo de primeira ordem, com elevados coeficientes de determinação e baixos valores de AIC. O excelente ajuste do modelo de primeira ordem indica afinidade dos microrganismos aeróbios pelo substrato. De fato, a ARC apresenta elevada

Tabela 2 - Valores médios dos parâmetros estimados pela modelagem da progressão de demanda bioquímica de oxigênio (DBO) (total e solúvel) exercida de cada água residuária agroindustrial (ARA).

\begin{tabular}{|c|c|c|c|c|c|c|}
\hline Modelo & Parâmetros & ARB & ARS & ARC & ARL & ARBF \\
\hline \multicolumn{3}{|c|}{ Progressão } & \multicolumn{4}{|c|}{$\mathrm{DBO}_{\mathrm{T}}$} \\
\hline \multirow{5}{*}{$\begin{array}{l}\text { Primeira } \\
\text { Ordem }\end{array}$} & $k^{\prime}\left(d^{-1}\right)$ & 0,563 & 0,165 & 0,308 & 0,395 & 0,414 \\
\hline & $R^{2}$ & 0,952 & 0,980 & 0,997 & 0,984 & 0,915 \\
\hline & RMSE & 0,298 & 0,602 & 0,049 & 0,489 & 0,127 \\
\hline & NRMSE & 7,085 & 4,054 & 1,430 & 3,622 & 6,967 \\
\hline & AIC & $-29,452$ & $-14,234$ & $-112,463$ & $-18,034$ & $-68,168$ \\
\hline \multirow{6}{*}{$\begin{array}{l}\text { Segunda } \\
\text { Ordem }\end{array}$} & $\mathrm{k}^{\prime \prime}\left(\mathrm{L} \mathrm{d}^{-1} \mathrm{~g}^{-1}\right)$ & 0,308 & 0,022 & 0,203 & 0,062 & 0,554 \\
\hline & $\mathrm{R}^{2}$ & 0,845 & 0,946 & 0,940 & 0,935 & 0,981 \\
\hline & RMSE & 0,534 & 0,982 & 0,225 & 1,001 & 0,060 \\
\hline & NRMSE & 12,673 & 6,608 & 9,539 & 7,413 & 3,319 \\
\hline & $\mathrm{AIC}$ & $-14,334$ & 1,403 & $-54,664$ & 2,020 & $-93,381$ \\
\hline & $\mathrm{DBO}_{\mathrm{UT}}\left(\mathrm{gO}_{2} \mathrm{~L}^{-1}\right)$ & 4,21 & 14,85 & 3,44 & 13,50 & 1,82 \\
\hline \multicolumn{3}{|c|}{ Progressão } & \multicolumn{4}{|c|}{$\mathrm{DBO}_{\mathrm{s}}$} \\
\hline \multirow{5}{*}{$\begin{array}{l}\text { Primeira } \\
\text { Ordem }\end{array}$} & $k^{\prime}\left(d^{-1}\right)$ & 0,232 & 1,013 & 0,175 & 0,346 & 0,435 \\
\hline & $\mathrm{R}^{2}$ & 0,956 & 0,986 & 0,987 & 0,970 & 0,973 \\
\hline & RMSE & 0,278 & 0,013 & 0,090 & 0,608 & 0,050 \\
\hline & NRMSE & 7,418 & 2,935 & 3,247 & 5,171 & 4,133 \\
\hline & $\mathrm{AIC}$ & $-31,322$ & $-136,191$ & $-89,385$ & $-11,950$ & $-99,708$ \\
\hline \multirow{6}{*}{$\begin{array}{l}\text { Segunda } \\
\text { Ordem }\end{array}$} & $\mathrm{K}^{\prime \prime}\left(\mathrm{L} \mathrm{d}^{-1} \mathrm{~g}^{-1}\right)$ & 0,114 & 6,166 & 0,127 & 0,060 & 0,882 \\
\hline & $\mathrm{R}^{2}$ & 0,7843 & 0,986 & 0,912 & 0,919 & 0,962 \\
\hline & RMSE & 0,527 & 0,013 & 0,238 & 1,001 & 0,060 \\
\hline & NRMSE & 14,077 & 2,853 & 10,848 & 8,518 & 4,930 \\
\hline & $\mathrm{AIC}$ & $-14,664$ & $-137,101$ & $-52,617$ & 2,023 & $-93,713$ \\
\hline & $\mathrm{DBO}_{\mathrm{US}}\left(\mathrm{gO}_{2} \mathrm{~L}^{-1}\right)$ & 3,74 & 0,45 & 2,78 & 11,75 & 1,22 \\
\hline
\end{tabular}

ARB: água residuária de abatedouro; ARS: água residuária de suinocultura; ARC: água residuária de cervejaria; ARL: água residuária de laticínios; ARBF: água residuária de beneficiamento de frutas para produção de sorvetes; $K^{\prime}$ : constante de desoxigenação de primeira ordem; $K^{\prime \prime}$ : constante de desoxigenação de segunda ordem; $R^{2}$ : coeficiente de determinação; RMSE: erro quadrado médio; NRMSE: erro quadrado médio normalizado; AIC: critério de informação de Akaike; DBO : DBO última total; $\mathrm{DBO}_{\text {us: }} \mathrm{DBO}$ última solúvel. biodegradabilidade aeróbia por ser composta majoritariamente de compostos orgânicos de fácil degradação microbiana, como carboidratos e resíduos de leveduras provenientes das operações envolvidas no processo de produção de cerveja (BAKARE; SHANBAGU; CHETTY, 2017). O modelo de primeira ordem também foi o que melhor se ajustou aos dados de $\mathrm{DBO}_{\mathrm{S}}$ exercida para todas as ARA, com exceção da ARS, com coeficiente de determinação ligeiramente maior $\left(\mathrm{R}^{2}=0,98\right)$ e menor valor de $\operatorname{AIC}(-137,101)$ para o modelo de segunda ordem.

Os resultados obtidos neste trabalho permitem concluir que o modelo de primeira ordem descreveu satisfatoriamente a progressão de DBO das ARA estudadas e também para as de composição bioquímica mais complexa (elevadas concentrações de sólidos suspensos e matéria orgânica na fração particulada - Tabela 1), como as ARB (em ambos os tipos de progressão de $\mathrm{DBO}$ ) e as ARS (na progressão de $\mathrm{DBO}_{\mathrm{T}}$ ), corroborando as ideias de autores clássicos que defendem o modelo de primeira ordem como mais adequado (MAY, 1971; STREETER; PHELPS, 1925).

Na progressão de $\mathrm{DBO}_{\mathrm{T}}$, observa-se, na Tabela 2, que o valor de $k$ da $\operatorname{ARB}\left(k^{\prime}=0,56 \mathrm{~d}^{-1}\right)$ foi o maior entre as ARA avaliadas, indicando que os microrganismos conseguem rapidamente consumir a fração biodegradável da matéria orgânica. Segundo Pereira, Paiva e Silva (2016) e Wang, Jena e Das (2018), a ARB tem elevada biodegradabilidade relacionada a compostos solúveis, alta concentração de macro e micronutrientes, tamponamento natural e uma pronta disponibilidade de biomoléculas que podem ser facilmente oxidadas por microrganismos aeróbios em condições propícias, o que explica o resultado obtido para essa ARA. Contudo a oxidação dessas biomoléculas promove toxicidade a níveis agudos e crônicos, sendo necessário o tratamento desse efluente antes de sua disposição em corpos hídricos (PEREIRA; PAIVA; SILVA, 2016).

O valor de $k$ depende das características da matéria orgânica da ARA, da temperatura e da presença de substâncias inibidoras. De forma geral, quanto maior o valor de $k$, maior a taxa de consumo de material orgânico e de depleção de oxigênio. Conforme Matos et al. (2014), quanto mais facilmente assimilável for a matéria orgânica presente no meio, maior será o valor de $k$, desde que haja a ausência de substâncias inibidoras.

O menor valor de $k^{\prime}$ foi observado para a ARS $\left(k^{\prime}=0,16 \mathrm{~d}^{-1}\right)$, indicando que a taxa de consumo de matéria orgânica ocorreu de forma mais lenta até atingir o valor da $\mathrm{DBO}_{\mathrm{u}}\left(14,85 \mathrm{~g} \mathrm{~L}^{-1}\right)$. Esse resultado pode ser explicado pela ocorrência elevada de materiais de difícil estabilização na ARS in natura, como dejetos suínos e matéria orgânica particulada em geral $\left(\mathrm{DQO}_{\mathrm{P}} / \mathrm{DQO}_{\mathrm{T}}=0,80\right)$, bem como pela presença de inibidores tóxicos aos microrganismos envolvidos no tratamento, como resíduos de hormônios e medicamentos (DING et al., 2017; MATOS et al., 2014). 
Na progressão de $\mathrm{DBO}_{\mathrm{s}}$, o valor de $k$ ' foi o maior para a ARS $\left(k^{\prime}=1,01 \mathrm{~d}^{-1}\right)$, apesar de o modelo de segunda ordem ter se ajustado ligeiramente melhor à progressão de $\mathrm{DBO}_{\mathrm{S}}$ dessa ARA. No experimento realizado com ARS filtrada, a maior parte dos materiais de difícil estabilização é eliminada, e, portanto, o k'é maior nessas condições.

Em seus estudos, Marske e Polkowski (1972) chegaram a um consenso de uma relação entre o $k^{\prime}$ e os modelos de primeira e de segunda ordem: se $k^{\prime}$ for menor do que $0,34 \mathrm{~d}^{-1}$, o modelo de primeira ordem descreve melhor a progressão de DBO exercida; se estiver entre 0,34 e $0,45 \mathrm{~d}^{-1}$, ambos os modelos podem ser utilizados; e, caso seja maior do que $0,45 \mathrm{~d}^{-1}$, recomenda-se adotar o de segunda ordem.

As faixas estipuladas pelos autores concordam parcialmente com os resultados obtidos para as ARA estudadas. Para a progressão de $\mathrm{DBO}_{\mathrm{T}}$, ARS e ARC apresentaram $k^{\prime}<0,34 \mathrm{~d}^{-1}$ com elevados coeficientes de determinação (0,98 e 0,99, respectivamente); ARL e ARBF apresentaram $k^{\prime}$ entre 0,34 e $0,45 \mathrm{~d}^{-1}$, com elevado coeficiente de determinação apenas para ARL $\left(R^{2}=0,98\right)$. A ARBF foi mais bem descrita pelo modelo de segunda ordem, vistos o maior coeficiente de determinação $\left(\mathrm{R}^{2}=0,98\right)$ e os menores valores das funções de erro.

A ARB obteve $k^{\prime}>0,45 \mathrm{~d}^{-1}$ e, apesar disso, foi significativamente mais bem descrita pelo modelo de primeira ordem, já que o coeficiente de determinação do ajuste ao modelo de segunda ordem foi insatisfatório $\left(R^{2}=0,84\right)$. Dessa forma, pode-se concluir que as faixas estipuladas pelos autores são consistentes, mas que não podem ser aplicadas para todos os tipos de ARA, vista a grande variabilidade desses efluentes, sendo necessário que se avaliem os coeficientes de determinação e as funções de erro em cada modelagem.

Uma análise similar pode ser feita em relação à progressão de $\mathrm{DBO}_{s}$ : para ARB e ARC, foram encontrados $k^{\prime}<0,34 \mathrm{~d}^{-1}$, e o ajuste se deu com elevados coeficientes de determinação $(0,95$ e 0,98 , respectivamente) ao modelo de primeira ordem; ARL e ARBF apresentaram $k^{\prime}$ entre 0,34 e $0,45 \mathrm{~d}^{-1}$, e ambas com ajuste satisfatório ao modelo de primeira ordem, coeficientes de determinação de 0,97 e menor correlação com o modelo de segunda ordem pelos valores mais baixos do coeficiente de determinação $\left(R^{2}=0,92\right.$ para ARL e $\mathrm{R}^{2}=0,96$ para ARBF). Para a ARS, o valor de $k$ ' encontrado foi maior do que $0,45 \mathrm{~d}^{-1}$, e, de fato, o modelo de segunda ordem se ajustou de forma mais satisfatória aos dados experimentais, com coeficiente de determinação de 0,88 .

Ressalta-se que os valores de $k^{\prime}$ e de $k^{\prime \prime}$ não podem ser comparados de forma direta, visto que apresentam unidades diferentes. Portanto, coeficientes de primeira ordem não podem ser aplicados em equações de segunda ordem e vice-versa (MATOS et al., 2017). Neste trabalho, optou-se pela discussão detalhada do $k$ ' pelo melhor ajuste do modelo de primeira ordem aos dados experimentais das ARA estudadas. São escassos os estudos de modelagem para a obtenção dos coeficientes relacionados à progressão de $\mathrm{DBO}$ de $\mathrm{ARA}$. Os resultados de alguns estudos estão na Tabela 3.

Observa-se, por meio da Tabela 3, que o valor determinado por Fia et al. (2007) para a constante de desoxigenação de primeira ordem $\left(k^{\prime}=0,13 \mathrm{~d}^{-1}\right) \mathrm{da}$ água residuária da lavagem e despolpamento de frutos do cafeeiro (ARFC) é inferior ao estimado para a ARBF da indústria de sorvetes utilizada neste trabalho, que apresentou $k^{\prime}=0,41 \mathrm{~d}^{-1}$. Esse resultado permite inferir que a ARBF possui maior teor de compostos biodegradáveis de disponibilidade facilitada do que a ARA utilizada por Fia et al. (2007).

A ARFC é rica em matéria orgânica, proteínas, minerais e fibras, sendo também constituída de compostos tóxicos de difícil degradação, como compostos fenólicos e tanino, o que dificulta a biodegradabilidade aeróbia desse substrato (FIA et al., 2011; JUNG; KIM; SHIN, 2010). Apesar de esses resíduos serem oriundos do beneficiamento de frutos, as diferenças em suas composições físico-químicas influenciam na DBO exercida no tempo e na velocidade de consumo da matéria orgânica por microrganismos aeróbios.

O valor de $k$ ' obtido na presente contribuição para a ARS $\left(0,16 \mathrm{~d}^{-1}\right)$ situou-se como um valor intermediário aos relatados em Fia, Matos e Fukunaga (2004) e Matos et al. (2014), enquanto o valor de $k^{\prime}$ para a ARL $\left(0,40 \mathrm{~d}^{-1}\right)$ apresentou-se superior. Isso pode ser justificado pela variabilidade natural das características físico-químicas da ARA.

Esse resultado permite inferir que a generalização ou padronização de coeficientes de desoxigenação para ARA se torna incerta e imprecisa. Contudo isso não desqualifica o mérito da pesquisa realizada, pois os resultados obtidos contribuem como uma base de dados

Tabela 3 - Valores típicos do coeficiente de desoxigenação de primeira ordem da progressão de demanda bioquímica de oxigênio (DBO), obtidos em temperatura de $20^{\circ} \mathrm{C}$, para diferentes águas residuárias agroindustriais.

\begin{tabular}{|c|c|c|}
\hline Água residuária agroindustrial & $k^{\prime}\left(d^{-1}\right)$ & Referências \\
\hline Suinocultura & 0,24 & \multirow{3}{*}{$\begin{array}{c}\text { Fia, Matos } \\
\text { e Fukunaga } \\
\text { (2004) }\end{array}$} \\
\hline Laticínios & 0,18 & \\
\hline Avicultura & 0,21 & \\
\hline Suinocultura & $0,07-0,41$ & \multirow{2}{*}{$\begin{array}{l}\text { Matos et al. } \\
\quad(2014)\end{array}$} \\
\hline Laticínios & $0,13-0,31$ & \\
\hline Beneficiamento de frutos do cafeeiro & 0,13 & Fia et al. (2007) \\
\hline Abatedouro & 0,56 & \multirow{5}{*}{ Este Trabalho } \\
\hline Suinocultura & 0,16 & \\
\hline Cervejaria & 0,31 & \\
\hline Laticínios & 0,40 & \\
\hline Beneficiamento de frutos & 0,41 & \\
\hline
\end{tabular}

k': constante de desoxigenação de primeira ordem. 
científicos aplicáveis para a simulação de processos de degradação da matéria orgânica em condições aeróbias. Dessa forma, visando ao projeto de estações de tratamento de águas residuárias em condições aeróbias ou ao estudo de autodepuração de corpos hídricos, torna-se ideal a realização de ensaios de progressão de DBO para o levantamento das constantes cinéticas com base no efluente que será objeto de tratamento.

Matos et al. (2014) avaliaram o binômio tempo-temperatura na DBO exercida ao longo do tempo obtendo $k^{\prime}$ de $0,07 \mathrm{~d}^{-1}$ e $0,13 \mathrm{~d}^{-1}$, respectivamente, para ARS e ARL, valores abaixo dos encontrados por Fia, Matos e Fukunaga (2004). No caso da ARS, os autores atribuíram esses resultados à presença de substâncias inibidoras (hormônios e medicamentos). Todavia a causa mais provável foi a ocorrência do fenômeno de nitrificação, que pode interferir de forma decisiva na determinação da DBO e, portanto, na determinação do $k$ '.

$\mathrm{Na}$ demanda nitrogenada (demanda de segundo estágio), as formas reduzidas de nitrogênio (presentes previamente nas amostras ou disponibilizadas após a quebra das cadeias carbono-nitrogênio) são oxidadas por microrganismos autotróficos em uma série de etapas, até se converterem em nitratos (UDEIGWE; WANG, 2010). Assim, por demandar oxigênio dissolvido, a nitrificação é interferente na quantificação da DBO de amostras de água.

Nesse sentido, eventuais processos de nitrificação (demanda de segundo estágio) são capazes de prejudicar o ajuste dos modelos aos dados e à obtenção da $\mathrm{DBO}_{\mathrm{U}}$ e de $k$ e, portanto, as estimativas reais da eficiência de tratamento em sistemas biológicos aeróbios. Segundo Green e Ward (2011), a demanda de segundo estágio iniciar-se-ia entre o quinto e o oitavo dia de incubação, nos quais a depleção de oxigênio começa a se estabilizar e, posteriormente, volta a acelerar em uma tendência assintótica, chegando a um valor maior.

O fenômeno da nitrificação é observado visualmente na progressão de DBO no tempo pela mudança na tendência da curva da demanda de primeiro estágio, na qual há uma sobrelevação. Além disso, o surgimento de picos de DBO e o mau ajuste dos modelos aos dados podem indicar a oxidação de nitrogênio reduzido (MATOS et al., 2017).

É possível indicar, por meio da Figura 1, algumas das progressões nas quais a nitrificação pode ter influenciado na progressão de DBO exercida das ARA avaliadas, sobretudo a partir do quinto dia de experimento, como nas Figuras $1 \mathrm{~A}\left(\mathrm{ARB}, \mathrm{DBO}_{\mathrm{T}}\right)$ e $1 \mathrm{D}\left(\mathrm{ARS}, \mathrm{DBO}_{\mathrm{S}}\right)$. Conforme dados expressos na Tabela 1, essas duas ARA são as que apresentam maiores teores de nitrogênio em sua composição $(502,9$ e 723,4 mg- $\mathrm{N} \mathrm{L}^{-1}$, respectivamente) entre as ARA estudadas, o que justificaria o comportamento das curvas. Além disso, de acordo com a Figura 1, percebe-se o ótimo ajuste do modelo de primeira ordem aos dados experimentais de cada ARA, sobretudo para a ARC na progressão de $\mathrm{DBO}_{\mathrm{T}}$, as exceções são na progressão de $\mathrm{DBO}_{\mathrm{T}}$ e $\mathrm{DBO}_{\mathrm{S}}$ para a
ARBF e para a ARS, respectivamente, as quais se ajustaram de forma mais satisfatória ao modelo de segunda ordem, como mencionado anteriormente.

Em seus estudos, Matos et al. (2014) determinaram novamente o coeficiente de desoxigenação desconsiderando os dados de DBO obtidos além do oitavo dia, já que a atividade das bactérias nitrificantes é maior a partir desse dia de incubação. Os autores obtiveram resultados mais satisfatórios do que na primeira modelagem (anteriormente citada), apresentando $k^{\prime}$ de $0,41 \mathrm{~d}^{-1}$ para ARS e $0,31 \mathrm{~d}^{-1}$ para ARL, os quais são mais consistentes com os dados cinéticos encontrados neste estudo (Tabela 3).

Esses resultados sugerem que o uso de agentes antinitrificantes, como a 2-cloro-6-triclorometil piridina (TCMP), nos dias em que ocorrer a demanda de segundo estágio, poderia auxiliar na obtenção de dados de $\mathrm{DBO}$ de primeiro estágio mais assertivos para essas ARA, eliminando a interferência do consumo de oxigênio pela nitrificação e auxiliando no melhor ajuste do modelo aos dados (MATOS et al., 2017). Contudo, o não uso de agentes antinitrificantes não desqualifica o mérito do resultado.

Por meio da progressão de DBO exercida (Figura 1) no tempo, também se determinou a $\mathrm{DBO}_{\mathrm{U}}{ }^{20^{\circ} \mathrm{C}} \mathrm{T}$ para as $\mathrm{ARB}, \mathrm{ARS}, \mathrm{ARC}, \mathrm{ARL}$ e $\mathrm{ARBF}$, as quais foram de 4.210, 14.850, 3.440, 13.500 e $1.820 \mathrm{mgO}_{2}$ $\mathrm{L}^{-1}$, respectivamente (Tabela 2). Esses valores se referem à concentração de matéria orgânica biodegradável inicial ou à quantidade de oxigênio dissolvido (OD) requerido para a total degradação do material orgânico presente na água residuária, sendo maior que os valores de $\mathrm{DBO}_{5}{ }^{20^{\circ} \mathrm{C}} \mathrm{T}$ de 4.100, 7.979, 2.295, 11.875 e $1.620 \mathrm{mgO}_{2} \mathrm{~L}^{-1}$, encontrados para as ARB, ARS, ARC, ARL e ARBF, respectivamente.

Dado o período de incubação de apenas 5 dias, o parâmetro $\mathrm{DBO}_{5}^{20^{\circ} \mathrm{C}} \mathrm{T}$ subestima a quantidade de matéria orgânica biodegradável realmente presente na ARA, bem como a real quantidade de oxigênio necessária para estabilizá-la durante o processo aeróbio, comprometendo a precisão de dimensionamentos de reatores aeróbios ou a veracidade dos resultados obtidos em estudos de modelagem da qualidade da água em rios, como autodepuração, impossibilitando a determinação da sua capacidade de recuperação para a verificação do atendimento à legislação ambiental.

Nesse contexto, tanto os coeficiente de desoxigenação (Tabelas 2 e 3) quanto os estudos de DBO exercida no tempo (Figura 1) com os valores de $\mathrm{DBO}_{\mathrm{U}}{ }^{20^{\circ} \mathrm{C}}$ (Tabela 2) apresentados neste trabalho se mostram indispensáveis para estudos de cinética de desoxigenação em águas residuárias, autodepuração de corpos hídricos, projetos de lagoas de estabilização, reatores aeróbios ou demais trabalhos com as referidas ARA que necessitem realizar balanços de massa em termos de matéria orgânica biodegradável aerobiamente para previsão de eficiências. 

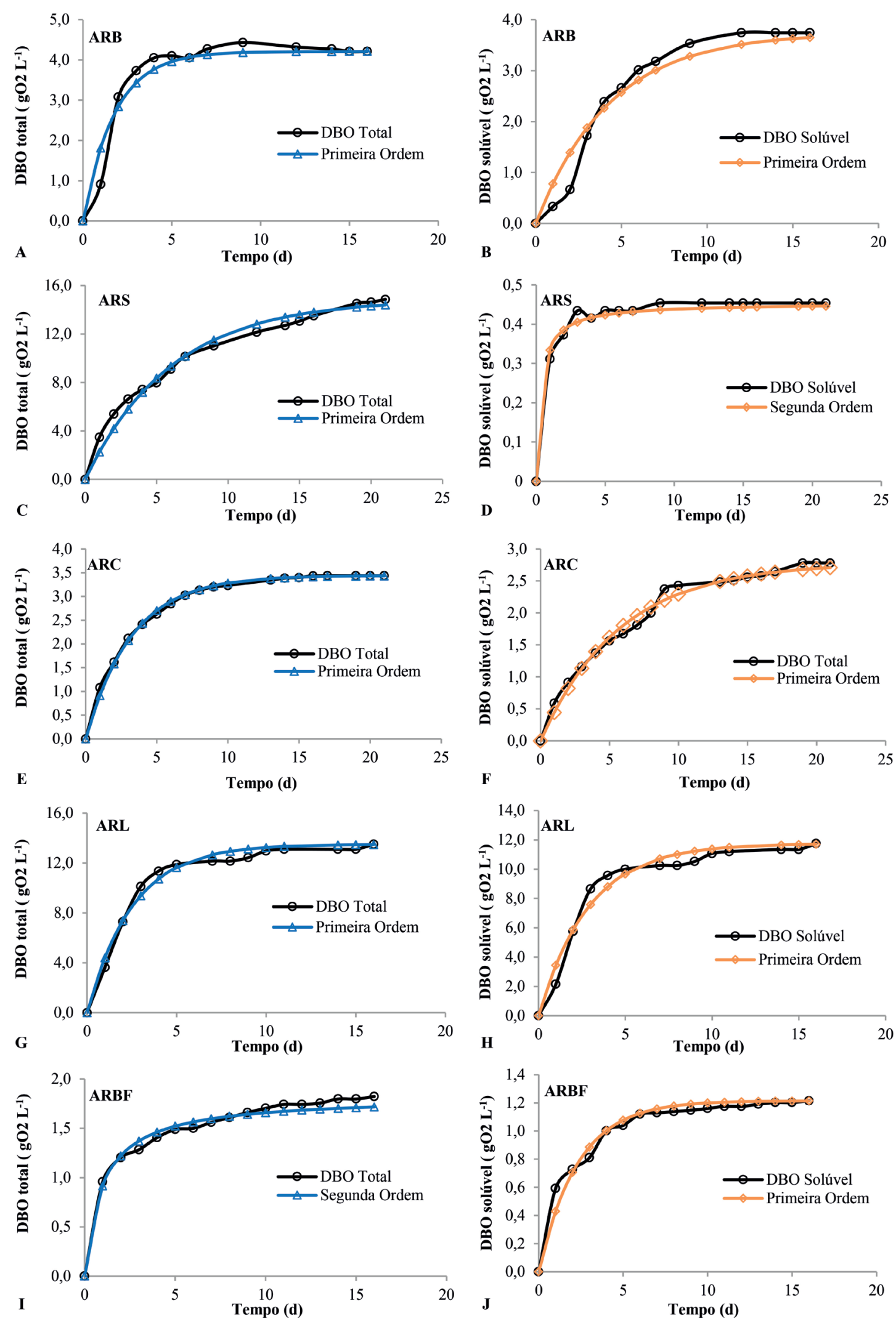

ARB: água residuária de abatedouro; ARS: água residuária de suinocultura; ARC: água residuária de cervejaria; ARL: água residuária de laticínios; ARBF: água residuária de beneficiamento de frutas para produção de sorvetes.

Figura 1 - Curvas de progressão de demanda bioquímica de oxigênio (DBO) exercida (total e solúvel) e curva gerada pelo modelo que melhor descreveu a progressão de DBO para cada uma das águas residuárias agroindustriais (ARA). 


\section{CONCLUSÕES}

A cinética de biodegradação aeróbia das ARA avaliadas ajustou-se melhor ao modelo de primeira ordem em termos de DBO total e solúvel. Na progressão de DBO total, o maior coeficiente de desoxigenação de primeira ordem $(k$ ) foi o da água residuária de abatedouro $\left(0,56 \mathrm{~d}^{-1}\right)$ e o menor foi o da água residuária de suinocultura $\left(0,16 \mathrm{~d}^{-1}\right)$. Os coeficientes de desoxigenação determinados no presente trabalho mostraram-se representativos, podendo ser utilizados para a simulação de processos de degradação da matéria orgânica em condições aeróbias.

\section{REFERÊNCIAS}

ABREU, P.; PEREIRA, E.L.; CAMPOS, C.M.M.; NAVES, F.L. (2O13) Photocatalytic Oxidation Process $\left(\mathrm{UV} / \mathrm{H}_{2} \mathrm{O}_{2} / \mathrm{ZnO}\right)$ in the treatment and sterilization of dairy wastewater. Acta Scientiarum Technology, v. 35, n. 1, p. 75-81. https://doi.org/10.4025/actascitechnol.v35i1.11132

ADRIAN, D.D.; SANDERS, T.G. (1998) Oxygen sag equation for second-order BOD decay. Water Research, v. 32, n. 3, p. 840-848. https://doi.org/10.1016/S0043-1354(97)00259-5

AKAIKE, H. (1998) Information theory and an extension of the maximum likelihood principle. In: PARZEN, E.; TANABE, K.; KITAGAWA, G. (orgs.). Selected Papers of Hirotugu Akaike. Nova York: Springer. p. 199-213.

AMERICAN PUBLIC HEALTH ASSOCIATION (APHA). (2012) Standard Methods for the Examination of Water and Wastewater. 22. ed. Washington, D.C.: APHA.

BAKARE, B.F.; SHABANGU, K.; CHETTY, M. (2017) Brewery wastewater treatment using laboratory scale aerobic sequencing batch reactor. South African Journal of Chemical Engineering, v. 24, p. 128-134. https://doi.org/10.1016/j.sajce.2017.08.001

BHATT, A.H.; ALTOUQI, S.; KARANJEKAR, R.V.; HOSSAIN, M.D.S.; CHEN, V.P.; SATTLER, M.S. (2016) Preliminary regression models for estimating first-order rate constants for removal of BOD and COD from landfill leachate. Environmental Technology \& Innovation, v. 5, p. 188-198. https://doi.org/10.1016/j.eti.2016.02.002

BOSONA, T.; GEBRESENBET, G. (2018) Life cycle analysis of organic tomato production and supply in Sweden. Journal of Cleaner Production, v. 196, p. 635-643. https://doi.org/10.1016/j. jclepro.2018.06.087

CENTRO DE ESTUDOS AVANÇADOS EM ECONOMIA APLICADA (CEPEA). EscolaSuperior de Agricultura LuizdeQueiróz. Universidade de São Paulo. PIB do Agronegócio Brasil (Dezembro/2017). São Paulo: CEPEA, 2018. 19 p. Disponivel em: <https://www.cepea.esalq. usp.br/upload/kceditor/files/Relatorio\%2OPIBAGRO\%2OBrasil_ DEZEMBRO CNA.pdf>. Acesso em: 13 jan. 2019.

CHAN, Y.J.; CHONG, M.F.; LAW, C.L.; HASSELL, D.G. (2009) A review on anaerobic-aerobic treatment of industrial and municipal wastewater. Chemical Engineering Journal, v. 155, n. 1-2, p. 1-18. https://doi.org/10.1016/j.cej.2009.06.041

CHENG, D.L.; NGO, H.H.; GUO, W.S.; CHANG, S.W.; NGUYEN, D.D.; KUMAR, S.M. (2019) Microalgae biomass from swine wastewater and its conversion to bioenergy. Bioresource Technology, v. 275 p. 109-122. https://doi.org/10.1016/j.biortech.2018.12.019

CHENG, D.L.; NGO, H.H.; GUO, W.S.; CHANG, S.W.; NGUYEN, D.D. KUMAR, S.M.; DU, B.; WEI, Q.; WEI, D. (2O18) Problematic effects of antibiotics on anaerobic treatment processes in swine wastewater Bioresource Technology, v. 263, p. 642-653. https://doi.org/10.1016/j. biortech.2018.05.010

CORSINO, S.F.; BIASE, A.; DEVLIN, T.R.; MUNZ, G.; TORREGROSSA, M.; OLESZKIEWICZ, J.A. (2017) Effect of Extended Famine Conditions on Aerobic Granular Sludge Stability in the Treatment of Brewery Wastewater. Bioresource Technology, v. 226, p. 150-157. https://doi org/10.1016/j.biortech.2016.12.026

CRISTOVÃO, R.O.; PINTO, V.M.S.; MARTINS, R.J.E.; LOUREIRO, J.M.; BOAVENTURA, R.A.R. (2016) Assessing the influence of oil and grease and salt content on fish canning wastewater biodegradation through respirometric tests. Journal of Cleaner Production, v. 127 p. 343-351. https://doi.org/10.1016/j.jclepro.2016.04.057

DANESHVAR, E.; ZARRINMEHR, M.J.; KOUTRA, E.; KORNAROS, M.; FARHADIAN, O.; BHATNAGAR, A. (2019) Sequential cultivation of microalgae in raw and recycled dairy wastewater: Microalgal growth, wastewater treatment and biochemical composition Bioresource Technology, v. 273, p. 556-564. https://doi.org/10.1016/j. biortech.2018.11.059

DEMIREL, B.; OROK, M.; HOT, E.; ERKIŞI, S.; ALBÜKREK, M.; ONAY, T.T. (2013) Recovery of biogas as a source of renewable energy from ice-cream production residues and wastewater. Environmental Technology, v. 34, n. 13-14, p. 2099-2104. https://doi.org/10.1080/09 593330.2013.774055

DING, W.; CHENG, S.; YU, L.; HUANG, H. (2017) Effective swine wastewater treatment by combining microbial fuel cells with flocculation. Chemosphere, v. 182, p. 567-573. https://doi.org/10.1016/j. chemosphere.2017.05.006

EMILIANO, P.C.; VIVANCO, M.J.F.; MENEZES, F.S.; AVELAR, F.G. (2009) Foundations and Comparison of Information Criteria: Akaike and Bayesian. Revista Brasileira de Biometria, v. 27, n. 3, p. 394-411.

ENITAN, A.M.; ADEYEMO, J.; KUMARI, S.; SWALAHA, F.M.; BUX, F. (2015) Characterization of Brewery Wastewater Composition. International Scholarly and Scientific Research \& Innovation, v. 9, n. 9, p. 1073-1076. 
FIA, R.; MATOS, A.T.; FUKUNAGA, D.C. (2004) Cinética de degradação da matéria orgânica de águas residuárias de origem animal. In: CONGRESSO BRASILEIRO DE ENGENHARIA AGRICOLA, São Paulo, 2004. Anais...

FIA, R.; MATOS, A.T.; LUIZ, F.A.R.; PEREIRA, P.A. (2007) Coeficientes de degradação da matéria orgânica de água residuária da lavagem e descascamento dos frutos do cafeeiro em condições anóxica e aeróbia. Engenharia na Agricultura, Viçosa, v. 15, n. 1, p. 45-54.

FIA, R.; MATOS, A.T.; SCHUERY, F.C.; TEODORO, P.E.P.; LUIZ, F.A.R. (2011) Desempenho de filtros anaeróbios no tratamento de águas residuárias da lavagem e descascamento dos frutos do cafeeiro. Engenharia na Agricultura, v. 19, n. 1, p. 62-71. https://doi.org/10.13083/ reveng.v19i1.277

FIA, R.; PEREIRA, E.L.; FIA, F.R.L.; EMBOABA, D.G.; GOMES, E.M. (2O15) Start-up of anaerobic reactors for slaughterhouse wastewater treatment. Engenharia Agrícola, v. 35, n. 2, p. 331-339. https://doi. org/10.1590/1809-4430-Eng.Agric.v35n2p331-339/2015

FOOD AND AGRICULTURE ORGANIZATION OF THE UNITED NATIONS (FAO). (2017) The State of Food and Agriculture: Leveraging Food Systems for Inclusive Rural Transformation. Roma: FAO. 181 p. Disponível em: <http://www.fao.org/3/a-i7658e. pdf>. Acesso em: 13 jan. 2019.

GREEN, B.; WARD, G.H. (2011) Ultimate biochemical oxygen demand in semi-intensively managed shrimp pond waters. Aquaculture, v. 319, n. 1-2, p. 253-261. https://doi.org/10.1016/j.aquaculture.2011.06.031

HILARIO GARCIA, A.L.; MATZENBACHER, C.A.; SANTOS, M.S.; PRADO, L.; PICADA, J.N.; PREMOLI, S.M.; CORREA, D.S.; NIEKRASZEWICZ, L.; DIAS, J.F.; GRIVICICH, I.; SILVA, J. (2017) Genotoxicity induced by water and sediment samples from a river under the influence of brewery effluent. Chemosphere, v. 169, p. 239-248. https://doi. org/10.1016/j.chemosphere.2016.11.081

JUNG, K.W.; KIM, D.-H.; SHIN, H.S. (2010) Continuous fermentative hydrogen production from coffee drink manufacturing wastewater by applying UASB reactor. International Journal of Hydrogen Energy, v. 35, n. 24, p. 13370-13378. https://doi.org/10.1016/j. ijhydene.2009.11.120

MARSKE, D.M.; POLKOWSKI, L.B. (1972) Evaluation of methods for estimating biochemical oxygen demand parameters. Journal (Water Pollution Control Federation), v. 44, n. 10, p. 1987-2000. Disponível em: <www.jstor.org/stable/25037634>. Acesso em: 15 jan. 2019

MATOS, M.P.; BORGES, A.C.; MATOS, A.T.; SILVA, E.F.; MARTINEZ, M.A. (2014) Effect of binominal time-temperature in obtaining biochemical oxygen demand of different wastewaters. Engenharia Agricola, v. 34, n. 2, p. 332-340. http://dx.doi.org/10.1590/S010069162014000200014

MATOS, M.P.; BORGES, A.L.; MATOS, A.T.; SILVA, E.F.:; MARTINEZ, M.A. (2017) Modelagem da progressão da DBO obtida na incubação de esgoto doméstico sob diferentes temperaturas. Engenharia Sanitária e Ambiental, v. 22, n. 5, p. 821-828. http://dx.doi.org/10.1590/ s1413-41522017101993
MAY, T.G. (1971) The effect of temperature on the ultimate BOD of sewage. Tese (Doutorado) - Northwestern University, Evanston.

MENKITI, M.C.; ANEKE, M.C.; EJIKEME, P.M.; ONUKWULI, O.D.; MENKITI, N.U. (2014) Adsorptive treatment of brewery effluent using activated Chrysophyllum albidium seed shell carbon. SpringerPlus, v. 3, p. 213. https://dx.doi.org/10.1186\%2F2193-1801-3-213

MOTTERAN, F.; PEREIRA, E.L.; CAMPOS, C.M.M. (2013a) Characterization of an acidification and equalization tank (AET) operating as a primary treatment of swine liquid effluent. Brazilian Archives of Biology and Technology, v. 56, n. 3, p. 485-494. https:// doi.org/10.1590/S1516-89132013000300017

MOTTERAN, F.; PEREIRA, E.L.; CAMPOS, C.M.M. (2013b) The behavior of an anaerobic baffled reactor (ABR) as the first stage in the biological treatment of hog farming effluents. Brazilian Journal of Chemical Engineering, v. 30, n. 2, p. 299-310. https://doi. org/10.1590/S0104-66322013000200008

OKE, I.A.; AKINDAHUNSI, A.A. (2005) A statistical evaluation of methods of determining BOD rate. Journal of Applied Sciences Research, v. 1, n. 2, p. 223-227.

PEREIRA, E.L.; CAMPOS, C.M.M.; MOTTERAN, F. (2013) Physicochemical study of $\mathrm{pH}$, alkalinity and total acidity in a system composed of Anaerobic Baffled Reactor in series with Upflow Anaerobic Sludge Blanket reactor in the treatment of pig farming wastewater. Acta Scientiarum Technology, v. 35, n. 3, p. 477-483. https://doi.org/10.4025/actascitechnol.v35i3.14069

PEREIRA, E.L.; CAMPOS, C.M.M.; MOTERANI, F.; OLIVEIRA NETO, A.M. (2011) Eficiência de um sistema de reatores anaeróbios no tratamento de efluentes líquidos de suinocultura. Acta Scientiarum Technology, v. 33, n. 3, p. 287-293. https://doi.org/10.4025/ actascitechnol.v33i3.7941

PEREIRA, E.L.; PAIVA, T.C.B.; SILVA, F.T. (2O16) Physico-chemical and Ecotoxicological Characterization of Slaughterhouse Wastewater Resulting from Green Line Slaughter. Water, Air \& Soil Pollution, v. 227, n. 6, p. 199. https://doi.org/10.1007/s11270-016-2873-4

RAJAB, A.R.; SALIM, M.R.; SOHAILI, J.; ANUAR, A.N.; SALMIATI; LAKKABOYANA, S.K. (2017) Performance of integrated anaerobic/ aerobic sequencing batch reactor treating poultry slaughterhouse wastewater. Chemical Engineering Journal, v. 313, p. 967-974. https:// doi.org/10.1016/j.cej.2016.10.144

RIBEIRO, R.; VON ATZINGEN, G.V.; LIMA, F.; OKAMOTO, V.H.T.; ARCE, A.I.C.; TOMMASO, G.; COSTA, E.J.X. (2017) Real-time control system based on the values of derivative of the redox potential aiming nitrogen removal in a Sequencing Batch Reactor applied in treating dairy wastewater. Water, Air \& Soil Pollution, v. 228, p. 228-231. https://doi.org/10.1007/s11270-017-3401-x

SIBIL, R.; BERKUN, M.; BEKIROGLU, S. (2014) The comparison of different mathematical methods to determine the BOD parameters, a new developed method and impacts of these parameters variations on the design of WWTPs. Applied Mathematical Modelling, v. 38, n. 2, p. 641-658. https://doi. org/10.1016/j.apm.2013.07.013 
STREETER, H.W.; PHELPS, E.B. (1925) A study of the natural purification of the Ohio River. Washington, D.C.: Public Health Service. (Public Health Bulletin, v. 146.)

TAMBURINI, E.; PEDRINI, P.; MARCHETTI, M.G.; FANO, E.A.; CASTALDELLI, G. (2015) Life cycle based evaluation of environmental and economic impacts of agricultural productions in the Mediterranean area. Sustainability, v. 7, p. 2915-2935. https:// doi.org/10.3390/su7032915

UDEIGWE, T.K.; WANG, J.J. (2010) Biochemical oxygen demand relationships in typical agricultural effluents. Water, Air \& Soil Pollution, Dordrecht, v. 213, p. 237-249. https://doi.org/10.1007/ s11270-010-0381-5

WANG, S.; JENA, U.; DAS, K.C. (2018) Biomethane production potential of slaughterhouse waste in the United States. Energy
Conversion and Management, v. 173, p. 143-157. https://doi. org/10.1016/j.enconman.2018.07.059

XIAO, S.; HU, S.; ZHANG, Y.; ZHAO, X.; PAN, W. (2O18) Influence of sewage treatment plant effluent discharge into multipurpose river on its water quality: A quantitative health risk assessment of Cryptosporidium and Giardia. Environmental Pollution, v. 233, p. 797-805. https://doi.org/10.1016/j.envpol.2017.11.010

YANG, D; DENG, L;:ZHENG, D; WANG, L.; LIU, Y.(2O16)Separation of swine wastewater into different concentration fractions and its contribution to combined anaerobic e aerobic process. Journal of Environmental Management, v. 168, p. 87-93. https://doi.org/10.1016/j.jenvman.2015.11.049

ZANONI, A.E. (1967) Waste water deoxygenation at diferent temperatures. Water Research, v. 1, n. 8-9, p. 543-566. https://doi. org/10.1016/0043-1354(67)90038-3 